\title{
Síndrome de Miller Fisher tratado con plasmaféresis durante el embarazo: reporte de un caso y revisión de la literatura
}

\section{Miller Fisher syndrome treated with plasmapheresis during pregnancy: Case report and review of the literature}

Julián Andrés Ángel-Páez, M.D. ${ }^{1}$; Silvana Hurtado-Bugna, M.D. ${ }^{2}$; Rafael Leonardo Aragón-Mendoza, M.D. ${ }^{3}$; Marcela Altman-Restrepo, M.D. ${ }^{4}$; Ivonne Jeannette DíazYamal, M.D. ${ }^{5}$; Gabriel Adolfo Centanaro-Meza, Ph.D. ${ }^{6}$

Recibido: 28 de septiembre de 2020/Aceptado: 22 de abril de 2021

\section{RESUMEN}

Objetivo: reportar el caso de una paciente gestante con síndrome de Guillain-Barré (SGB) presentado en la variante denominada síndrome de Miller Fisher (SMF), y realizar una revisión en torno al diagnóstico, tratamiento y pronóstico de esta variedad de SGB durante la gestación.

Materiales y métodos: se presenta el caso de una gestante de 27 semanas con síndrome de Miller Fisher, quien fue tratada con plasmaféresis en un hospital militar de referencia, con evolución satisfactoria a los 15 días y continuación normal del

* Correspondencia: Julián Andrés Ángel Páez, Servicio de Ginecología y Obstetricia, Hospital Militar Central, transversal 3ra. No. 49-02, Bogotá (Colombia).julianangell17@gmail.com

1. Médico residente Ginecología y Obstetricia, Universidad Militar Nueva Granada, Bogotá (Colombia).

2. Médico residente Ginecología y Obstetricia, Universidad Militar Nueva Granada, Bogotá (Colombia).

3. Médico ginecobstetra, especialista Medicina Materno Fetal, Hospital Militar Central, Bogotá (Colombia).

4. Médico ginecobstetra, especialista Medicina Materno Fetal, Hospital Militar Central, Bogotá (Colombia)

5. Médico ginecobstetra, especialista en Medicina Reproductiva, Hospital Militar Central, Bogotá (Colombia). Docente titular Universidad Militar Nueva Granada, Bogotá (Colombia).

6. Médico, especialista en Neurología Clínica, doctor en Bioética, Hospital Militar Central, Bogotá. (Colombia). Docente titular Universidad Militar Nueva Granada, Bogotá, Colombia. embarazo, parto a las 38 semanas con recién nacido sano. Se realizó una búsqueda bibliográfica en bases de datos electrónicas: Medline vía PubMed, Lilacs, SciELO, ScienceDirect, Ovid, con los términos "Embarazo", "Síndrome de Miller Fisher", "Síndrome de Guillain-Barré”. Se incluyeron cohortes, series y reportes de casos de mujeres gestantes con síndrome de Miller Fisher; se extrajo información sobre los métodos diagnósticos, el tratamiento utilizado y el pronóstico materno y perinatal. La búsqueda se hizo en junio 2020, sin restricción por fecha, pero sí por tipo de idioma (español e inglés). Resultados: se identificaron 423 títulos, tres estudios cumplieron los criterios de inclusión, los tres correspondieron a reportes de caso. Todos los casos mostraron seropositividad para antigangliósidos GQ1b positivos; en ningún caso hubo alteración imagenológica. Dos pacientes recibieron inmunoglobulina intravenosa y la tercera paciente se dejó en observación. Hasta el momento no se documentan complicaciones obstétricas.

Conclusión: existen pocos casos reportados de SMF durante la gestación, el diagnóstico se basa en el examen clínico; el tratamiento con inmu- 
noglobulina IV representa la alternativa utilizada con mayor frecuencia. En el caso presentado se utilizó la plasmaféresis. Se desconoce el impacto de la variedad del síndrome de Miller Fisher sobre el curso normal de la gestación y sobre los resultados perinatales a largo plazo. Se requieren más estudios que aborden el diagnóstico, el tratamiento y el pronóstico de esta entidad.

Palabras clave: embarazo, síndrome de Miller Fisher, síndrome de Guillain-Barré.

\section{ABSTRACT}

Objective: To report the case of pregnant woman with Guillain-Barré syndrome (GBS) presenting as the Miller Fisher variant, and to review the literature on the diagnosis, treatment and prognosis of this GBS variant during gestation.

Material and methods: Pregnant woman presenting at 27 weeks of gestation with Miller Fisher syndrome (MFS), treated in a military referral hospital with a satisfactory course after 15 days, continuation of normal pregnancy and delivery of a healthy neonate at 38 weeks. A search of the literature was conducted in the Medline via PubMed, Lilacs, SciELO, ScienceDirect and Ovid databases using the terms "Pregnancy," "Miller Fisher syndrome," "GuillainBarré syndrome”. Cohorts, case series and case reports of pregnant women with MFS were included. Data on diagnostic methods, treatment and maternal and perinatal prognosis were extracted. The search was made on June 2020, with no restriction by date, but restriction by language (Spanish and English).

Results: Overall, 423 titles were identified, three studies met the inclusion criteria, the three of them corresponding to case reports. All cases were found to be seropositive for anti-GQ1b ganglioside antibodies. No imaging abnormalities were found in any of the cases. Two patients received IV immunoglobulin and the third patient was kept under observation. No obstetric complications have be documented so far.

Conclusion: There are few cases of MFS reported during pregnancy. Intravenous immunoglobulin is the most frequently used treatment option. Plasmapheresis was used in the case presented here. The impact of the Miller Fisher variant on the normal course of gestation and on long-term perinatal outcomes is unknown. Further studies that look into the diagnosis, treatment and prognosis of this condition are required. .

Keywords: Pregnancy, Miller Fisher syndrome, Guillain-Barré syndrome.

\section{INTRODUCCIÓN}

El síndrome de Guillain-Barré (SGB) es una enfermedad neurológica progresiva, caracterizada por la aparición de debilidad ascendente y simétrica (1), y es la principal causa de parálisis aguda (2). La frecuencia está entre 0,8 y 2,6 x 100.000 personas/año, y se incrementa con la edad; es más frecuente en hombres (3). En la actualidad se describen tres variantes del SGB: neuropatía motora axonal aguda (AMAN), neuropatía axonal motor-sensorial aguda (AMSAN) y el síndrome de Miller Fisher (SMF) (4). Esta última variedad se caracteriza por la presencia de la triada de oftalmoplejía, ataxia y arreflexia; sin embargo, se han descrito diferentes formas de presentación (5). En cuanto a su etiología, una importante proporción de los casos de SGB se asocian a un evento infeccioso, principalmente de índole respiratorio o gastrointestinal (4) incluyendo el virus del Zika (6); también se han descrito otros factores asociados como la vacuna de la influenza (7), la cirugía y el embarazo (4). Respecto al SMF se ha descrito la asociación a un fenómeno autoinmune dado por la presencia de anticuerpos antigangliósidos GQ1b (8).

El diagnóstico del SGB es básicamente clínico con un cuadro agudo progresivo de parálisis motora principalmente, con hiporreflexia- (4). Para el diagnóstico del SGB posvacunal y el SMF se han utilizado los criterios diagnósticos de la Colaboración Brighton (9). Para el SMF son muy útiles los anticuerpos antigangliósidos GQ1b, en la fase aguda de la enfermedad (primera semana), con una sensibilidad descrita del $92 \%$ y una especificidad del $97 \%$ (8). Dentro de los hallazgos paraclínicos sugestivos de SGB se describe también la disociación albúmino-citológica en el líquido cefalorraquídeo, distintiva de la entidad a 
partir de la segunda semana desde la instauración del cuadro clínico, y el uso de pruebas de conducción nerviosa (10). El tratamiento del SGB se basa en la formulación de inmunoglobulina endovenosa o plasmaféresis $(11,12)$; en cuanto al pronóstico, cerca del $20 \%$ presentan secuelas neurológicas a largo plazo y la mortalidad puede ser de hasta un $5 \%$ en la población general (13).

Respecto a su presentación durante el embarazo, su incidencia es similar a la observada en la población general (14) y se ha descrito que se presenta mayor frecuencia en la segunda mitad de la gestación y en el puerperio (15). En cuanto al tratamiento, este es similar al de la mujer no gestante; respecto al pronóstico, se ha descrito una mortalidad materna esperada que varía entre el $3 \%$ (16) y el $10 \%$, con requerimiento de cuidados intensivos en el $35 \%$ de los casos (15).

El SGB es una entidad importante para el obstetra ya que el embarazo puede estar asociado a su aparición, además de estar asociado al virus del zika, infección viral que afectó a nuestra región en una epidemia en 2015 y 2016 (17), con especial repercusión en la salud perinatal (18). Por tanto, el obstetra debe pensar en esta entidad y sus diferentes variantes que incluyen el SMF, ya que su manejo en la gestante puede requerir un equipo interdisciplinario.

El objetivo de este estudio es describir el caso de una gestante con diagnóstico de síndrome de Miller Fisher asociado a parálisis periférica, y hacer una revisión de la literatura acerca del diagnóstico, el tratamiento y el pronóstico de esta variedad de presentación del SGB durante la gestación.

\section{PRESENTACIÓN DEL CASO}

Primigestante de 22 años de edad, con gestación de 27 semanas, que consultó por cuadro clínico de tres días de evolución al servicio de urgencias del Hospital Militar Central de Bogotá, institución de alta complejidad, que atiende población perteneciente a las fuerzas militares. La paciente presentaba cefalea frontal tipo peso, que se incrementaba con maniobras de Valsalva, de intensidad 9 sobre 10, asociado a fotofobia, fonofobia y escotomas. Con embarazo no planeado, sin control preconcepcional, con paraclínicos prenatales adecuados para la edad gestacional.

$\mathrm{Al}$ ingreso presentó tensión arterial 107/65 milímetros de mercurio ( $\mathrm{mmHg}$ ), frecuencia cardiaca de 63 latidos por minuto (lpm), frecuencia respiratoria de 16 respiraciones por minuto, Glasgow 15/15 y temperatura de 36 grados centígrados; mucosa oral húmeda, examen cardiopulmonar sin alteraciones, abdomen con altura uterina de 26 centímetros, feto único, fetocardia de 147 lpm, sin actividad uterina. $\mathrm{Al}$ examen neurológico se observó paciente alerta, orientada, lenguaje fluido, con fondo de ojo, campimetría, movimientos oculares y pares craneales normales; con midriasis plena no reactiva bilateral y ptosis (figura 1); no nistagmus; sensibilidad facial y simetría facial conservadas, úvula centrada, elevación simétrica del velo del paladar, reflejo nauseoso presente simétrico bilateral; fuerza $5 / 5$ en cuatro extremidades, reflejos musculotendinosos $++/++++$ en cuatro extremidades simétrico, respuesta plantar flexora bilateral, sensibilidad superficial y profunda conservada, sin dismetría ni disdiadococinesias, marcha sin alteraciones, sin signos de irritación meníngea. Se hospitalizó con diagnóstico de cefalea con signos de alarma. En los paraclínicos de ingreso se observó hemograma: 10.300 leucocitos x mcl; hemoglobina: 13,5 g/dl; hematocrito: 40,6\%, 348.000 plaquetas x mcl; PT (tiempo de protrombina): 10,1 segundos; PTT (tiempo parcial de tromboplastina): 23,9 segundos; creatinina: 0,62 mg/dL. Se solicitó resonancia magnética cerebral más venografía, la cual evidenció parénquima y sistema venoso cerebral sin alteraciones, con hallazgo incidental de mucocele (figura 2). Durante la hospitalización, en las siguientes 24 horas la afección pupilar bilateral se asocia a limitación para abducción, aducción, supraversión e infraversión del ojo izquierdo, oftalmoparesia que sugiere compromiso completo del III y VI par, disminución del esfuerzo tusígeno, arreflexia global, dismetría bilateral, ataxia y disautonomía, lo que configura el diagnóstico presuntivo de SMF. Se indicó la realización de anticuerpos antigangliósidos y 

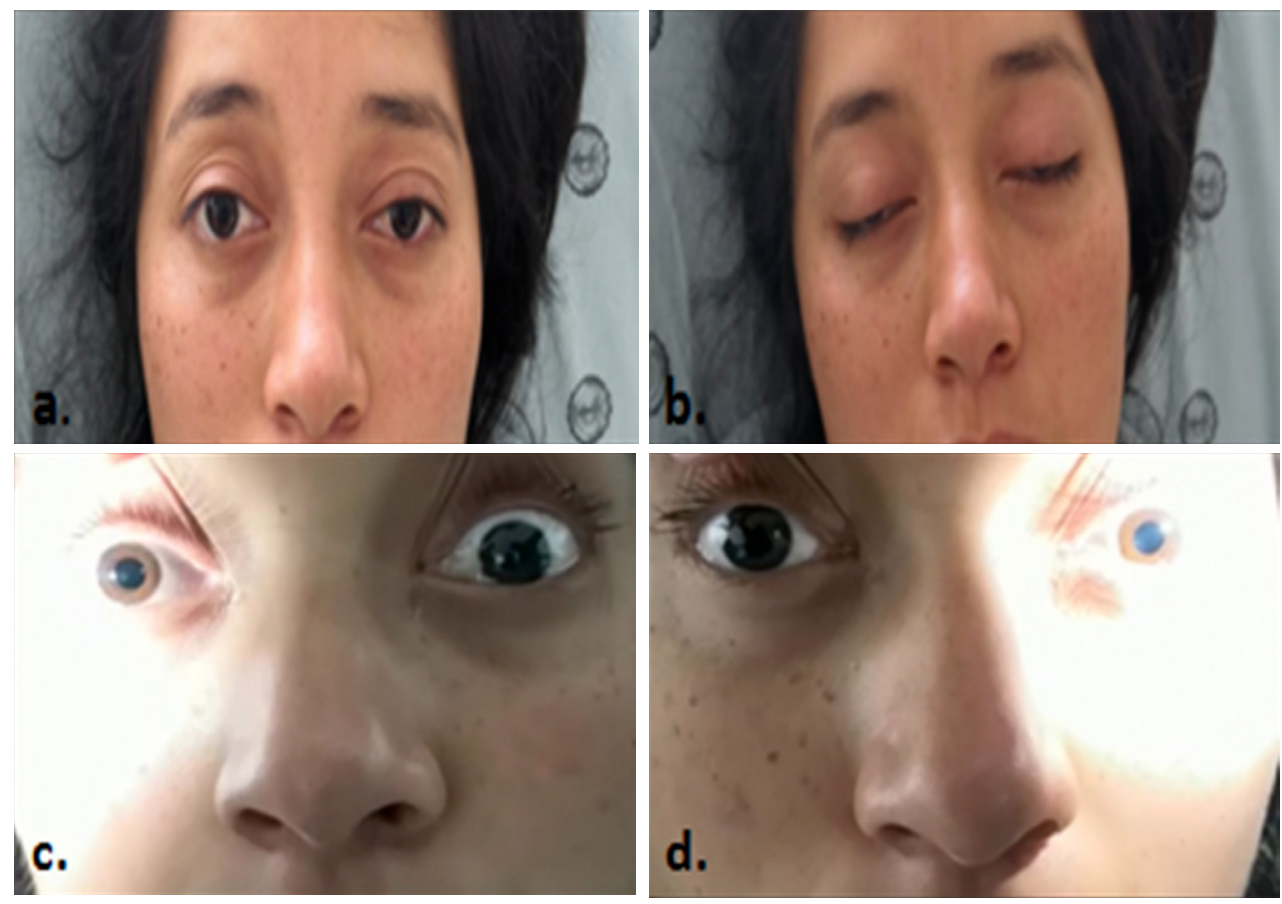

Figura 1. Examen ocular de ingreso de paciente con síndrome de Miller Fisher.

a. Se observa oftalmoplejía. b. Ptosis palpebral bilateral. c. y d. Ausencia de respuesta reflejo fotomotor con midriasis bilateral de $5 \mathrm{~mm}$

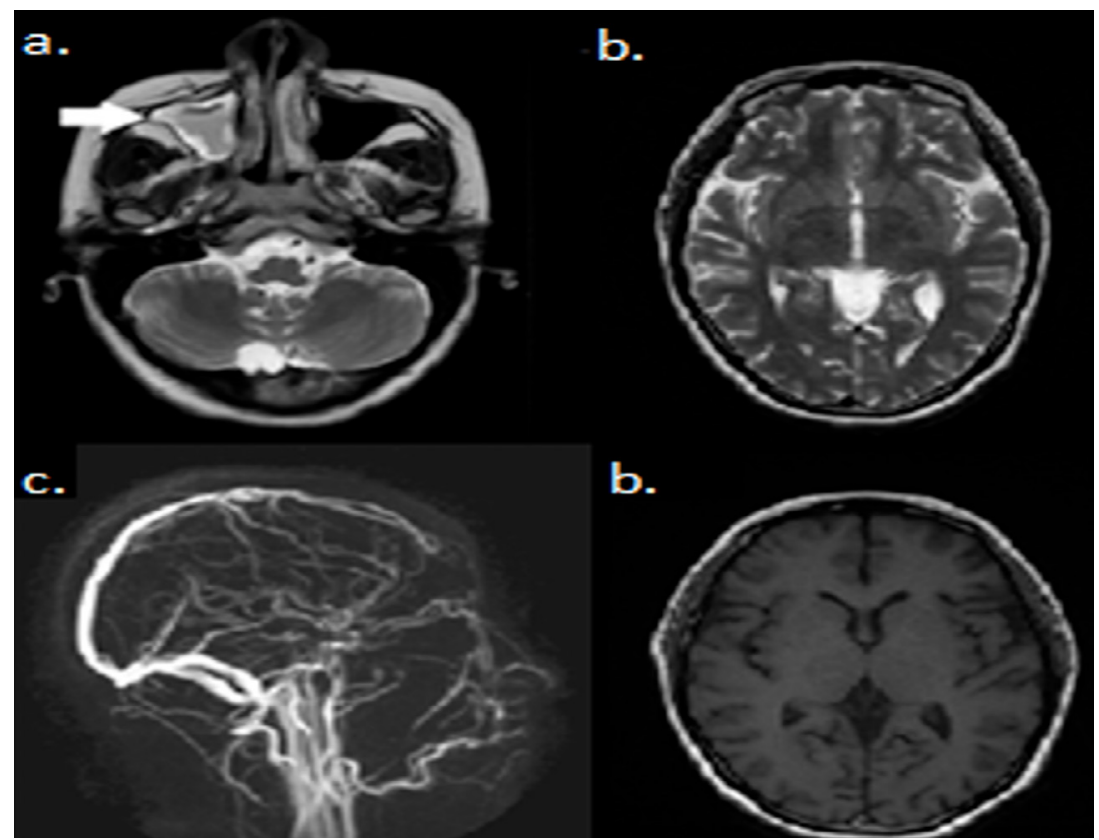

Figura 2. Resonancia magnética y venografía

a. Ocupación del seno maxilar derecho compatible con mucocele (flecha blanca). b. Parénquima cerebral normal. c. Venografía normal.

se trasladó a la unidad de cuidados intensivos (UCI) para vigilancia estricta. Se le realizó punción lumbar la cual mostró un líquido cefalorraquídeo transparente, glucosa: 58 (mg/dl) (glucosa central de $104 \mathrm{mg} /$ dl), proteínas de $15 \mathrm{mg} / \mathrm{dl}$, sin evidencia de leucoci- tos o bacterias a la tinción de Gram, sin disociación albumino-citológica. Los estudios electrofisiológicos de las cuatro extremidades documentaron adecuada velocidad de conducción; silencio en reposo y actividad de inserción adecuada con potencial de acción 
de la unidad motora (PAUM) de duración y amplitud normal. Ondas F y H con adecuada reproductividad y latencia. Durante las primeras 12 horas en UCI presentó un deterioro progresivo dado por aumento de sialorrea, disfagia, disartria y paresia de miembros inferiores, por lo cual se indicó tratamiento con plasmaféresis con albúmina, requiriendo cinco sesiones interdiarias, con un recambio plasmático terapéutico de volumen plasmático de $2.416 \mathrm{cc}$ (peso ideal 59 $\mathrm{kg}$ - talla 1,68 m), con $2.355 \mathrm{cc}$ de lactato de Ringer y 785 cc de albúmina al 5\%. Se realizó seguimiento diario clínico y de laboratorio y además se inició maduración pulmonar fetal con betametasona. Se realizó vigilancia del bienestar fetal con monitoreo fetal diario y durante las cinco terapias de recambio plasmático, junto a perfil biofísico interdiario. Durante las dos semanas posteriores a la intervención, la paciente mostró mejoría gradual de los síntomas, con resolución de la ataxia y la disfagia lo que, sumado a la existencia de bienestar fetal, permitió seguimiento ambulatorio.

Durante el cuidado ambulatorio se observó re-
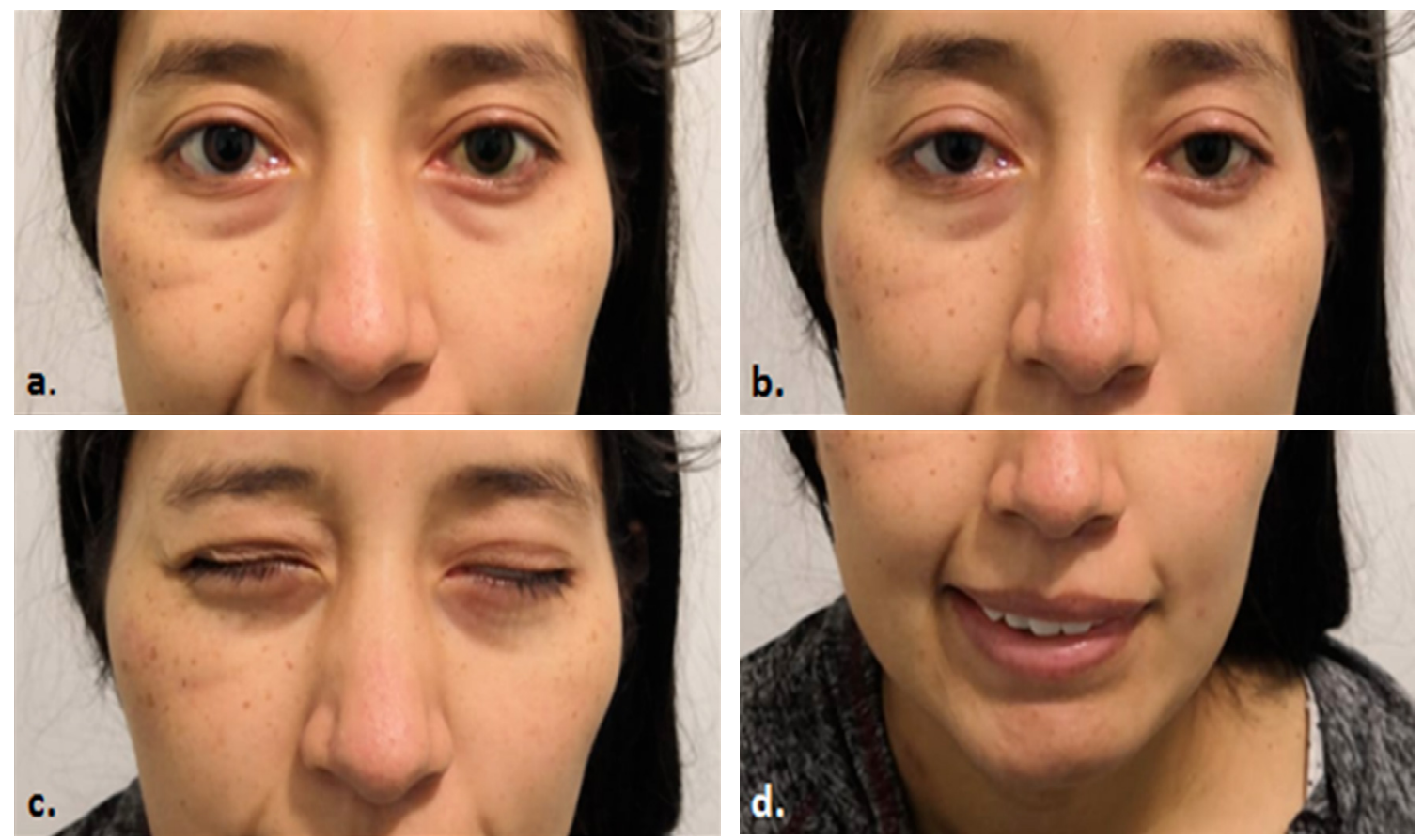

Figura 3. Control al egreso hospitalario de paciente con síndrome de Miller Fisher a y b. Resolución de oftalmoplejía. c. y d. Parálisis VII par craneal periférico izquierdo, limitación cierre ocular izquierdo, desviación comisura labial hacia la derecha. 


\section{MATERIALES Y MÉTODOS}

Con base en las preguntas ¿cómo se realiza el diagnóstico, el tratamiento y el pronóstico del síndrome de Miller Fisher durante el embarazo?, se llevó a cabo una búsqueda bibliográfica en las bases de datos electrónicas de: Medline vía PubMed, Lilacs, SciELO, ScienceDirect, Ovid, con los términos "Embarazo", "Síndrome de Miller Fisher" "Síndrome de Guillain-Barré". Se incluyeron estudios primarios como cohortes, series y reportes de caso, que presentaran mujeres gestantes con SMF que abordaran el diagnóstico, el tratamiento y el pronóstico de esta entidad durante la gestación. La pesquisa se hizo el 20 de junio de 2020, sin restricción por fecha, pero sí por tipo de idioma (español e inglés).

El proceso de selección se realizó en dos etapas. Inicialmente dos autores, de manera independiente, revisaron los artículos por título y resumen para verificar la presencia de los criterios de inclusión; en los que cumplían se obtuvo el texto completo para definir su aceptación definitiva. En caso de discrepancia se consultó a un tercer autor. Luego se realizó la extracción de los datos en un formulario específicamente diseñado para esto, las variables extraídas fueron: características del estudio: diseño, fecha y lugar de la presentación del caso; características de las pacientes: edad materna y edad gestacional al diagnóstico, presencia de comorbilidades; diagnóstico: clínico (síntomas y signo), hallazgos de laboratorio (en el líquido cefalorraquídeo, en detección de anticuerpos GQ1b); hallazgos en conducción nerviosa, resonancia magnética u otros estudios diagnósticos realizados; tratamiento (uso de plasmaféresis, corticoesteriodes, inmunoglobulina IV otros medicamentos); pronóstico: tiempo de resolución de los síntomas, resultados perinatales (complicaciones fetales, edad gestacional al parto, vía de parto, estado del recién nacido), complicaciones maternas. Los resultados se presentan de manera narrativa, de acuerdo con los dominios antes mencionados; además, se presenta una tabla resumen al final del documento.

\section{RESULTADOS}

Se encontraron 423 títulos, de los cuales se seleccionaron tres por cumplir los criterios de inclusión. Los estudios objeto de análisis correspondieron a tres reportes de caso con revisión de la literatura $(19,20,21)$ (Tabla 1).

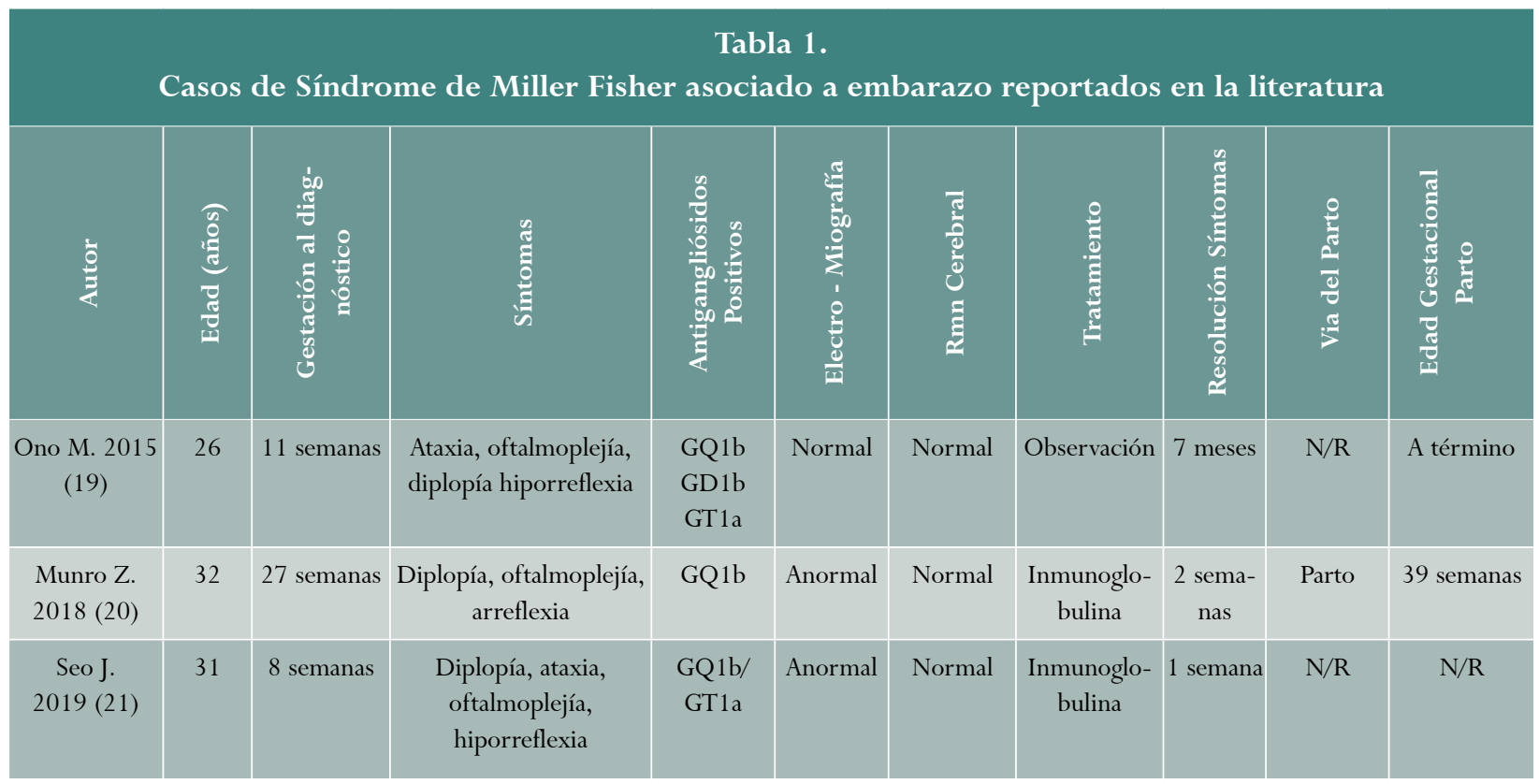

Fuente: elaboración propia.

$\mathrm{N} / \mathrm{R}$ : no registro. 


\section{Características de la población}

Edad materna: en los estudios recuperados, al momento del diagnóstico de SMF las pacientes cursaban la tercera (19) o cuarta década de la vida (20,21); respecto a la edad gestacional dos casos fueron diagnosticados durante el primer trimestre $(19,21)$ y el último, durante el tercer trimestre de la gestación (20).

Diagnóstico: se describe la hiporreflexia o arreflexia, acompañada de oftalmoplejía y diplopía en los tres reportes de casos $(19,20,21)$, con dos casos de ataxia como síntoma adicional $(19,21)$. Estudios paraclínicos: se observó la positividad de anticuerpos antigangliósidos en las tres pacientes, para GQ1b (19-21) o para GT1a (19,21); en ninguno de los casos se documentó disociación albuminocitológica en el estudio de líquido cefalorraquídeo. Estudios imagenológicos: la resonancia magnética (RM) fue la neuroimagen elegida en los tres casos, sin que se hubiese documentado algún hallazgo anormal (19-21). En cuanto a la conducción nerviosa, una paciente presentó neuropatía axonal motora (20) y en otra se detectó ausencia de Onda Hen extremidades inferiores asociada a arreflexia (21).

Tratamiento: dos pacientes recibieron inmunoglobulina intravenosa (IV) $(20,21)$, observando la resolución de los síntomas durante las dos siguientes semanas. La tercera paciente solo fue objeto de vigilancia clínica con resolución espontánea del cuadro clínico (19) luego de siete meses de seguimiento. En ninguno de los casos reportados se utilizó plasmaféresis, ni se requirió apoyo ventilatorio ni se describe el uso de corticoides para maduración pulmonar.

Pronóstico: en relación con el pronóstico fetal, en dos de los casos identificados la gestación culminó a término, con recién nacidos sin complicaciones perinatales $(19,20)$. El tercer caso no reportó el resultado perinatal (21) y solo uno informó la vía del parto, siendo este vaginal (20). Ninguno de los casos reportó la presencia de complicaciones maternas o fetales asociadas a la terapia o como consecuencia de la enfermedad.

\section{CONCLUSIONES}

Existen pocos casos reportados de SMF durante la gestación, el diagnóstico se basa en el examen clínico, el tratamiento con inmunoglobulina IV representa la alternativa utilizada con mayor frecuencia. En el caso presentado se utilizó la plasmaféresis. Se desconoce el impacto de la variedad del síndrome de Miller Fisher sobre el curso normal de la gestación y sobre los resultados perinatales a largo plazo. Se requieren más estudios que aborden el diagnóstico, el tratamiento y el pronóstico de esta entidad.

\section{REFERENCIAS}

1. Hughes RA, Cornblath DR. Guillain-Barre syndrome. Lancet. 2005;366:1653-16. https://doi.org/10.1016/ S0140-6736(05)67665-9

2. Malek E, Salameh J. Guillain-Barre Syndrome. Semin Neurol. 2019;39:589-95. https://doi. org/10.1055/s-0039-1693005

3. Sejvar JJ, Baughman AL, Wise M, Morgan OW. Population incidence of Guillain-Barré syndrome: A systematic review and meta-analysis. Neuroepidemiology. 2011;36(2):123-33. https://doi. org/10.1159/000324710.

4. Donofrio P. Guillain-Barré Syndrome. Continuum. 2017;23(5):1295-1309. https://doi.org/10.1212/ CON.0000000000000513.

5. Jung JH, Oh EH, Shin JH, Kim DS, Choi SY, Choi $\mathrm{KD}$, Choi JH. Atypical clinical manifestations of Miller Fisher syndrome. Neurol Sci. 2019;40(1):67-73. https://doi.org/10.1007/s10072-018-3580-2.

6. Watrin L, Ghawche F, Larre P, et al. GuillainBarre'syndrome (42 cases) occurring during a Zika virus outbreak in French Polynesia. Medicine (Baltimore). 2016;95(14):e3257. https://doi.org/10.1097/ MD.0000000000003257

7. Willison H, Bart J, Doorn P. Guillain-Barré syndrome. Lancet. 2016; 338:717-27. https://doi.org/10.1016/ S0140-6736(16)00339-1.

8. Patola M, Du Pasquier R, Schluep M, Regeniter A. Serum and CSF GQ1bantibodies in isolated ophthalmologic syndromes. Neurology. 2016;86:1780-4. https://doi.org/10.1212/WNL.0000000000002558 
9. Sejvar JJ, Kohl KS, Gidudu J, Amato A, Bakshi N, Baxter R, et al. Guillain-Barré syndrome and Fisher syndrome: Case definitions and guidelines for collection, analysis, and presentation of immunization safety data. Vaccine. 2011;29(3):599-612. https:// doi.org/10.1016/j.vaccine.2010.06.003.

10. Nishimoto Y, Odaka M, Hirata K, Yuki N. Usefulness of anti-GQ1b IgG antibody testing in Fisher syndrome compared with cerebrospinalfluid examination. J Neuroimmunol. 2004;148:200-5. https://doi. org/10.1016/j.jneuroim.2003.11.017

11. Elovaara I, Apostolski S, van Doorn P, Gilhus NE, Hietaharju A, Honkaniemi J, et al. EFNS guidelines for the use of intravenous immunoglobulin in treatment of neurological diseases. Eur J Neurol. 2008;15:893-908. https://doi.org/10.1111/j.14681331.2008.02246.x

12. Verboon C, Dan Doorn P, Jacobs B. Treatment dilemmas in Guillain-Barré syndrome. J Neurol Neurosurg Psychiatry. 2017;88(4):346-52. https://doi. org/10.1136/jnnp-2016-314862

13. Van den Berg B, Bunschoten C, van Doorn P, Jacobs B. Mortality in Guillain-Barre syndrome. Neurology. 2013;80(18):1650-4. https://doi.org/10.1212/ WNL.0b013e3182904fcc

14. Cheng Q, Jiang GX, Fredrikson S, Link H, de PedroCuesta J. Increased incidence of Guillain-Barré syndrome postpartum. Epidemiology. 1998;9:6014. https://doi.org/10.1097/00001648-19981100000006

15. Nelson LH, McLean WT Jr. Management of LandryGuillainBarré syndrome in pregnancy. Obstet Gynecol. 1985;65(suppl):25S-29S.

16. Kasaven LS, Basu C. Guillain-Barré syndrome in pregnancy: Successful multidisciplinary approach. Eur J Obstet Gynecol Reprod Biol. 2018:228:332. https:// doi.org/10.1016/j.ejogrb.2018.06.017.

17. Borchering RK, Huang AT, Mier-Y-Teran-Romero L, Rojas DP, Rodriguez-Barraquer I, Katzelnick LC, et al. Impacts of Zika emergence in Latin America on endemic dengue transmission. Nat Commun. 2019;10(1):1-9. https://doi.org/10.1038/s41467019-13628-x
18. Mora-Salamanca AF, Porras-Ramírez A, Restrepo FPH. Burden of disease due to microcephaly associated with the Zika virus in Colombia. Cad Saude Publica. 2020;36(11):e00215319. https://doi. org/10.1590/0102-311X00215319

19. Ono M, Sato H, Shirahashi M, Tomioka N, Maeda J, Watanabe K, et al. Clinical Features of Miller-Fisher Syndrome in Pregnancy. Case Rep Obstet Gynecol. 2015;2015:1-3. https://doi. org/10.1155/2015/840680.

20. Munro Z, Fernandez D. Clinical Reasoning: Acute onset of diplopia in pregnancy. Neurology. 2018;91:e180-e184. https://doi.org/10.1212/ WNL.0000000000005758.

21. Seo J, Kang M, Yoon B, Ji K, Oh S. Miller Fisher syndrome mimicking Wernicke encephalopathy during pregnancy. Ann Clin neurophysiol. 2019;21(1):53-56. https://doi.org/10.14253/acn.2019.21.1.53.

\section{CONTRIBUCIÓN DE LOS AUTORES}

Julián Andrés Ángel-Páez: elaboración del documento desde su concepción; diseño y adquisición de la información; revisión del contenido intelectual y aprobación de la versión enviada a proceso editorial; elaboración de fotos y tablas; participación en el diseño del material gráfico.

Silvana Hurtado-Bugna: elaboración del documento desde su concepción; diseño y adquisición de la información; revisión del contenido intelectual y aprobación de la versión enviada a proceso editorial; elaboración de fotos y tablas; participación en el diseño del material gráfico.

Rafael Leonardo Aragón-Mendoza: elaboración del documento desde su concepción, diseño y adquisición de la información; revisión del contenido intelectual y aprobación de la versión enviada a proceso editorial; elaboración de fotos y tablas; participación en el diseño del material gráfico.

Marcela Altman-Restrepo: elaboración del documento desde su concepción y diseño hasta la adquisición de la información, revisión del contenido intelectual y aprobación de la versión enviada a proceso editorial. 
Ivonne Jeannette Díaz-Yamal: elaboración del documento desde su concepción, diseño y adquisición de la información; revisión del contenido intelectual y aprobación de la versión enviada a proceso editorial. Gabriel Adolfo Centanaro-Meza: elaboración del documento desde su concepción y diseño hasta la adquisición de la información; revisión del contenido intelectual y aprobación de la versión enviada a proceso editorial.

Conflicto de intereses: ninguno declarado. 